\title{
Performance Assessment of Multi-State Systems with Critical Failure Modes: Application to the Flotation Metallic Arsenic Circuit
}

\author{
Seraphin C. Abou \\ Mechanical and Industrial Engineering Department, University of Minnesota Duluth, \\ 1305 Ordean Court, Duluth, MN 55812,
}

USA

\section{Introduction}

Conventional reliability theory assumes that sub-systems and the system itself usually have two different states: on (good, operating) or off (down, failed). Owing to this assumption, the structural function of the system is a binary function of binary variables, and the respective model is usually referred to as binary reliability system. However, for many engineering systems, the binary assumption may not be appropriate for describing the possible states that each of the components may experience throughout its life cycle [3], [6], [7], [8].

Precise evaluation of the state probability and performance rate of an element in some multi-state systems is difficult. Some reasons come from inaccuracy and insufficiency of data. In a broad sense, if an event or a kind of behavior of these components meets a predetermined criterion, whatever the criterion is, then we say it is a success. If the criterion is violated, then a failure occurs. Indeed, the accident literature is replete with examples, including the space shuttle Challenger (Vaughan, 1996), Three Mile Island (Chiles, 2002), the London Paddington train crash (Cullen, 2000) and Gulf of Mexico oil disaster among many others. In practice, nonlinearities may be present that are capable of significantly affecting systems performance. Typical phenomena resulting from the presence of nonlinearities include the onset of stable limit cycle oscillations determined by linear theory, or the existence of unstable limit cycles within the linear flutter boundary associated with a subcritical Hopf bifurcation.

Our motivation in this study is to carry out a theoretical study with practical relevance of nonlinear systems behavior and provide the performance analysis not in the time domain. Thus we assume that the function of safety is to locate and define the operational errors that allow accidents to occur. This function can be carried out in two ways: $i$.) by asking why accidents happen - searching for their root causes - and ii.) by assessing the performance levels of certain known effective engineering controls that are being utilized.

Causes of failure are diverse. They can be physical, human and logical or even financial [8]. Evidently, various kinds of criteria and factors can be taken into account to define what a failure means: structure, performance, cost and even subjective intention. However whatever a failure is, if effect of it tends to be critical, research on it becomes essential. In 
traditional reliability theory, the components of a system and the system itself are usually assumed to have two different states: on (good, operating) or off (down, failed). [10], [21], [23], [26].

For instance, in fluid control networks, a defective valve may be either "stuck-open" or "stuck-closed", in safety monitoring systems, a device will malfunction if it "fails to detect breakdown" or "initiates a false alarm" etc. A structure whose components experience two different modes of failure is usually referred to as three-state device. A natural extension of the three-state devices is easily developed by assigning to each component $m \geq 2$ failure modes. The resulting structure will then be called multistate system (MSS), [14], [16].

In complex MSSs consisting of $n$ elements, any element $j, 1 \leq j \leq n$ can have $k_{j}$ different states with corresponding performance rates (levels), which can be represented by the ordering set as follows, [13], [15]:

$$
g_{j}=\left\{g_{j, 1}, \ldots . . g_{j, i}, \ldots, g_{j, k_{j}}\right\}
$$

where $g_{j, i}$ is the performance rate (level) of the element $j$ in the state $i ; i \in\left\{1,2, \ldots ., k_{j}\right\}$.

The performance rate $G_{j}(t)$ of element $j$ at any instant $t \geq 0$ is a random variable that takes its values from $g_{j}: G_{j}(t) \in g_{j}$. Thus, the probabilities associated with different states for the element $j$ can be represented by a set: $p_{j}=\left\{p_{j, 1}, \ldots . p_{j, i}, \ldots, p_{j, k_{j}}\right\}$

The mapping $g_{j, i} \rightarrow p_{j, i}$ is usually called the probability mass function, [13], [14], [16].

There are two fundamental assumptions in the conventional multi-state system reliability theory: i.) each state probability of an element, which composed a multi-state system, can be fully characterized by probability measures; and ii.) the state performance rate (level) of an element, which composed a multi-state system, can be precisely determined.

One approach to carry out a theoretical study of nonlinear systems behavior is to perform the analysis in the time domain. However, in the literature review, a drawback with this is that though it can yield a complete picture of system behavior for a particular set of initial conditions, it may be inefficient in providing an overall picture of multi-state systems characteristics even for a single set of sub-system parameters. System availability is represented by a multi-state availability/stability function, which extends the binary-state availability. In fact, because modern systems are large scale systems with complex interactions between their elements, precipitating incidents and accidents may have long incubation periods, making identification of a leading error chain difficult. To satisfy the required multi-state system availability, the redundancy principle for each component or universal generating function has been used in [14], [19]. Tavakkoli et al. (2008) assumed a predetermined and fixed redundancy strategy for each subsystem which became an additional decision variable.

In this paper the procedures for the reliability estimation of a flotation circuit is based on the universal generating function (u-function) technique, which was introduced in [21], and proved to be very effective for the reliability evaluation of different types of multistate systems [17], [9] and high dimension combinatorial problems. The u-function extends the widely known ordinary moment generating function [18]. As a result, the concepts of relevancy, availability, coherency, and equivalence defined in this paper are used to characterize the properties of the MSSs. Without loss of the generality, in the former case we assumed that for the MSSs, these properties are strongly related to the stability concept depicted in fig.1. This assumption makes it the unique exception that has been disregarded in the literature review [7], [20], [26] for the MSSs performance assessment. 
Billinton R. \& Allan R., (Reliability evaluation of power systems, 1990) have developed a comparison between four different methods of the assessment of the large scale MSSs reliability and highlighted that the technique is fast enough to be used in complex problems where the search space is sizeable. Throughout, the states which obey the operational constraints and are located inside the polytope (i.e., the recovery zone) are called admissible states. This constitutes a tradeoff between software performance and reliability (particularly with regard to computational time). Moreover in this study, for practical importance, we paid particular attention to the system state's future trajectory so that, after a switch, it stays within the set of the admissible states and converge to the set point.

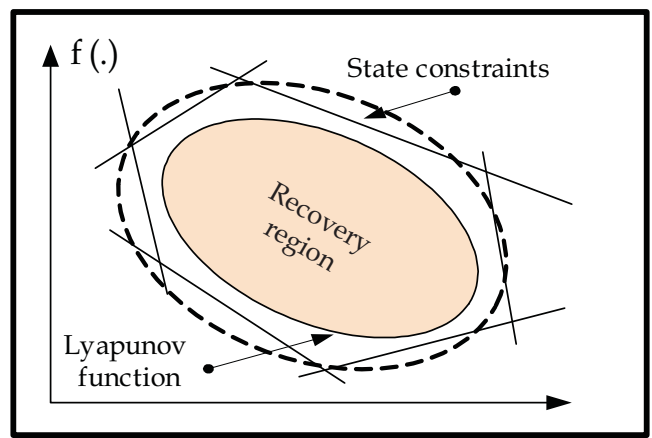

Fig. 1. State constraints and switching rule (Lyapunov function)

\section{Method for estimating system performance}

Performance is a key criterion in design, procurement, and usability of engineering system. In order to get the highest performance for the cost of a given system, an engineer needs, at least, a basic knowledge of performance evaluation terminology and techniques. An important problem in reliability theory is to determine the reliability of a complex system given the reliabilities of its components.

In real life the system and its components are capable of being in a whole range of states, varying from a perfect functioning state to states related to various levels of performance degradation to complete failure. Thus, the binary models are an oversimplification of the actual reality. This paper presents models and their applications in terms of reliability analysis to situations where the system can have whole range of states and all its components can also have whole range of multiple states. Generally a system has various levels of operational performance and hence the total system effectiveness measures should reflect all of these performance levels and their reliabilities. Evaluating design alternatives for linear systems, a number of methodologies are being used.

\subsection{Transmitted flow model of linear systems}

Let an expression of considerable importance of the design of a linear system be presented by the following block diagram, Fig. $2 . R$ is the referential input and $C$ is the output of the system.

Due to linear measurement characteristic of this system, the closed-loop block diagram, Fig. 2a.), could be reduced and replaced by an open loop block diagram, Fig. 2b.), with a new 
transfer function $G$ which gives the necessary and sufficient condition for stability in the frequency domain of the system.

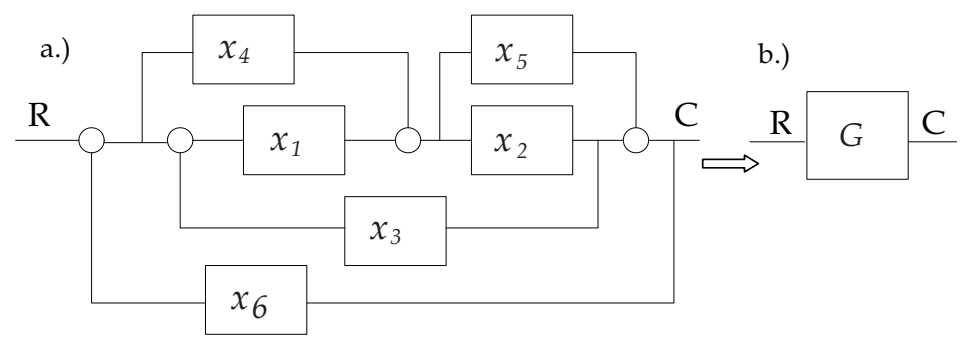

Fig. 2. Block diagram: a) Closed-loop representation b) Reduced block diagram in open-loop representation

The new function transfer $G$ is defined as follows:

$$
G=\frac{\left(1+\frac{x_{4}}{x_{1}}\right)\left(1+\frac{x_{1} x_{2}}{x_{1} x_{2} x_{3}}\right)\left(1+\frac{x_{5}}{x_{1}}\right)}{1+\left(1+\frac{x_{4}}{x_{1}}\right)\left(1+\frac{x_{1} x_{2}}{x_{1} x_{2} x_{3}}\right)\left(1+\frac{x_{5}}{x_{1}}\right) x_{6}}
$$

After some involved mathematical manipulations, the function $\wp(s)$ which defines an autonomous system whose characteristic equation maps the dynamics of the open loop system is obtained based on Nyquist stability criterion:

$$
\wp(s)=x_{1}^{3} x_{2} x_{3}+\left(x_{1}+x_{4}\right)\left(x_{1} x_{2} x_{3}+x_{1} x_{2}\right)\left(x_{1}+x_{5}\right) x_{6}
$$

Hence, performance levels of the given linear system could be assessed upon the response of $\wp(s)$ to a step stimulus. However, nonlinear systems don't offer such simple deductive analysis without losing information while involving simplest assumptions to facilitate their linearization. In the following section, we describe the technique used for evaluating complex systems availability and statistically expected performance while the nominal performance level and availability of their elements are given for open and closed modes.

\subsection{System structure and assumptions}

Nonlinear system control architectures can include static and dynamic feedback components as well as logic-based switching or discrete event elements. Such complex structures may arise as a matter of design choice or due to intrinsic constraints on the class of controllers that can be implemented.

The system under consideration is a mineral process shown in fig. 3. A wet grinding model has been analyzed with the objective of evaluating the effects of many variables on particle size reduction in continuous grinding processes.

Detailed phenomenological model that describes the charge behaviour has been developed and validated against real data [1]. Indeed, mineral processes present nonlinear/chaotic dynamics. The circuit consists of three variable velocity feeders, a main fixed velocity feeder, a ball mill, a sump, a variable velocity pump and a battery of hydro-cyclones. 


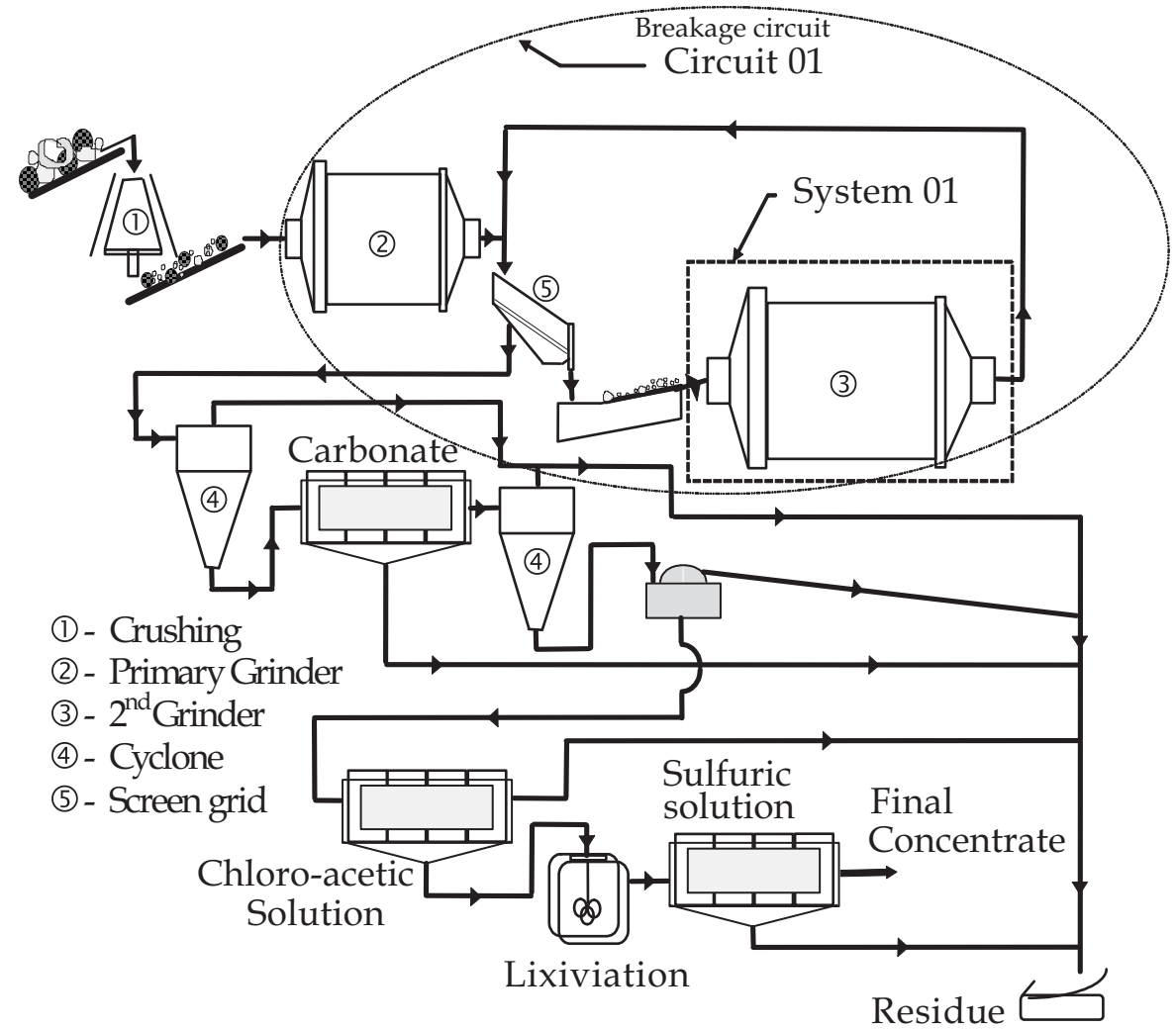

Fig. 3. Mineral processing

The fresh ore is transported towards the main feeder by the variable velocity feeders. Then it continues to the mill where water and the recirculated pulp are added. High performance level of the whole system determines the quality of the final product (the fineness of the grinded ore). This paper suggests reliability measures for complex systems. An important problem in reliability theory is to determine the reliability of a complex system given the reliabilities of its components. In real life, systems as shown in fig. 3 and their components are capable of being in a whole range of states, varying from a perfect functioning state to states related to various levels of performance degradation to complete failure.

\subsection{Interdependent systems}

Reliability theory distinguishes between independent and dependent systems. For dependent systems "component failures are in some way dependent" [13]. The term interdependent system has emerged, which we consider to be a subclass of dependent systems. For a system to be interdependent, mutual dependence in the sense of two-way causation among at least two components must be present. For example, if a "single failure" component fails and affects the second component, the system is dependent, but if the second component does not affect the main component, the system is not interdependent. Examples occur within aircraft systems, computer networks, fire protection, biological 
systems, etc... In [11] an airline baggage checking system example was illustrated. It is costly for the airline, but has limited impact if luggage transferred from other airlines is not checked.

Since the expected damage could not be precisely determined, assume $d_{e}$ as the expected damage and $d_{r}$ as the real impact on an interdependent system. The utility of a system of $n$ interdependent components are:

$$
\begin{array}{ll}
d_{e}=\sum_{i=1}^{n} \omega_{i} p_{i} ; & u=-\sum_{i=1}^{n}\left(\omega_{i} p_{i}+\kappa_{i} t_{i}\right) \\
d_{r}=\sum_{i=1}^{n} \varpi_{i} p_{i} ; & \mathrm{u}_{\mathrm{r}}=\sum_{i=1}^{n}\left(\varpi_{i} p_{i}-\delta_{i} T_{i}\right)
\end{array}
$$

An event may have a cumulative effect on the damage and the utility function. To account for interdependence between systems $i$ and $j$ the unreliable probability $p_{i}$ is generalized as follow:

$$
\begin{aligned}
& p_{i}=\left(\sum_{j=1}^{n} \ell_{i j}\left(t_{j}^{m_{j}}+T_{j}^{m_{j}}\right)\right)^{-1} \sum_{j=1}^{n} \ell_{i j} T_{j}^{m_{j}} \\
& \left\{\begin{array}{l}
i=j \rightarrow \ell_{i j}=1 \\
i \neq j \rightarrow 0 \leq \ell_{i j} \leq 1
\end{array}\right.
\end{aligned}
$$

where $t_{i}$ is the expected time at unit $\operatorname{cost} \kappa_{i}$ for the component $i ; \omega_{i}$ is the value of the component $i ; T_{i}$ is the duration of the damage at unit $\operatorname{cost} \delta_{i} ; \varpi_{i}$ is the damage value; $p_{i}$ the probability the system becomes dysfunctional; $\ell_{i j}$ interdependence between systems $i$ and $j$. Without interdependence $\ell_{i j}=0$ for all $i \neq j$

\subsubsection{Example of two interdependent systems}

As illustrated in Fig. 2, assume components 1 and 3 are interdependent. Then (6) becomes:

$$
\begin{aligned}
& p_{1}=\left(T_{1}^{m_{1}}+\ell T_{3}^{m_{3}}\right)\left(t_{1}^{m_{1}}+T_{1}^{m_{1}}+\ell\left(t_{3}^{m_{3}}+T_{3}^{m_{3}}\right)\right)^{-1} \\
& p_{3}=\left(T_{3}^{m_{3}}+\ell T_{1}^{m_{1}}\right)\left(t_{3}^{m_{3}}+T_{3}^{m_{3}}+\ell\left(t_{1}^{m_{1}}+T_{1}^{m_{1}}\right)\right)^{-1}
\end{aligned}
$$

$\ell_{31}=\ell_{13}=\ell$; and (4) and (5) becomes:

$$
\begin{aligned}
& d_{e 13}=\omega_{1} p_{1}+\omega_{3} p_{3} ; u_{13}=-\omega_{1} p_{1}-\omega_{3} p_{3}-\kappa_{1} t_{1}-\kappa_{3} t_{3} \\
& d_{r 13}=\varpi_{1} p_{1}+\varpi_{3} p_{3} ; u_{r 13}=\varpi_{1} p_{1}+\varpi_{3} p_{3}-\delta_{1} T_{1}-\delta_{3} T_{3}
\end{aligned}
$$

Appendix A solves the optimization problem when $m_{i}=1$, and $\ell_{31}=\ell_{13}=\ell$ to yield:

$$
t_{1}=\omega_{1}^{2} \varpi_{1}\left(\delta_{1}-\ell \delta_{3}\right)\left(\omega_{1} \delta_{1}+\varpi_{1} \kappa_{1}-\ell\left(\omega_{1} \delta_{3}+\varpi_{1} \kappa_{3}\right)\right)^{-2}+
$$




$$
\begin{aligned}
& -\ell \omega_{3}^{2} \varpi_{3}\left(\delta_{3}-\ell \delta_{1}\right)\left(\omega_{3} \delta_{3}+\varpi_{3} \kappa_{3}-\ell\left(\omega_{3} \delta_{1}+\varpi_{3} \kappa_{1}\right)\right)^{-2} \\
& p_{1}=\varpi_{1}\left(\kappa_{1}-\ell \kappa_{3}\right)\left(\omega_{1} \delta_{1}+\varpi_{1} \kappa_{1}-\ell\left(\omega_{1} \delta_{3}+\varpi_{1} \kappa_{3}\right)\right)^{-1}
\end{aligned}
$$

In case $\omega_{1}=\omega_{3} ; \varpi_{1}=\varpi_{3} ; \kappa_{1}=\kappa_{3}$ and $\delta_{1}=\delta_{3}$

$$
\left\{\begin{array}{l}
t_{1}=t_{3}=\omega_{1}^{2} \varpi_{1} \delta_{1}\left(\omega_{1} \delta_{1}+\varpi_{1} \kappa_{1}\right)^{-2} \\
p_{1}=p_{3}=\varpi_{1} \kappa_{1}\left(\omega_{1} \delta_{1}+\varpi_{1} \kappa_{1}\right)^{-1}
\end{array}\right.
$$

To illustrate how the framework in this chapter can be used to analyze a specific system performance, consider the mineral processing plant in fig.3. In mineral processing, electrochemical potential and related engineering control (e.g. flow control valves) is considered as an important parameter for controlling the recovery and selectivity of sulphide minerals during flotation. Consider, as an example, the flotation circuit of the process shown in fig.3. Flotation circuit illustrated in fig. 4 has been used in solid/solid separation applications using stable froths to recover the mineral particles. Flotation can be incorporated with wastewater-treatment schemes in the following ways: As a unit process for removing contaminants not separated by other processes or as a unit process for sludge thickening.

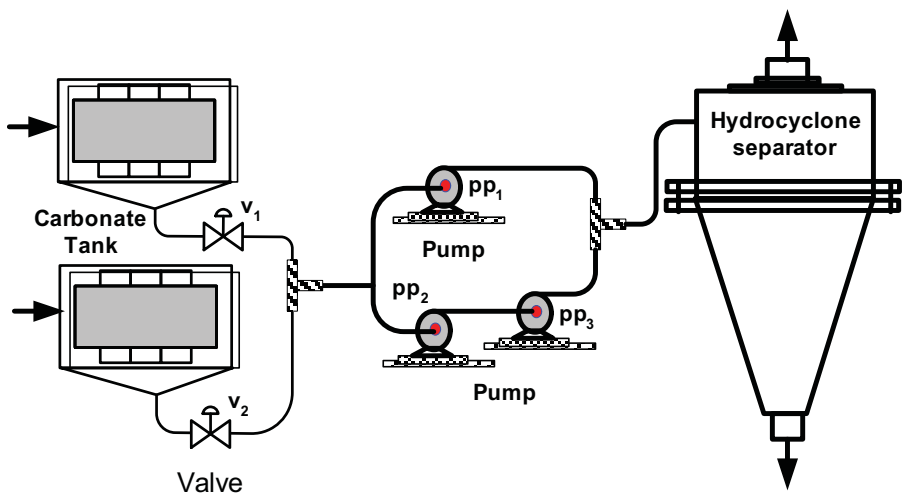

Fig. 4. Flotation circuit

The operating condition of the circuit in fig.4 is as follows: The minimum flow required for successful operation corresponds to the full capacity of one tank or one pump or one pipe. Any elementary component of the system (a tank, a pump or a pipe) is considered to have the following two states: i.) No flow; ii.) Full flow. These same states are considered to apply also for any subsystem. Each of the three pump-pipe subsystems can be modeled as two states homogeneous coherent (HC) system. We assume that these systems are multi-state systems with two failure modes (S2FM).

In the proposed technique applied to reliability analysis, components are characterized by two states: an up-state and a down-state (failure). We explore the possibility of studying system reliability, by modeling each component with a multi-state system approach, [13], [25]. Hence we focus on S2FM. The procedure of the reliability measures is based on the use 
of a universal generation function (UGF), [22]. Systems with two failure modes consist of devices, which can fail in either of two modes. For instance, servo-valves in the flotation circuit, Fig. 4, can not only fail to close when commanded to close but can also fail to open when commanded to open. In this circuit, we consider components consisting of different elements characterized by nominal performance level in each mode.

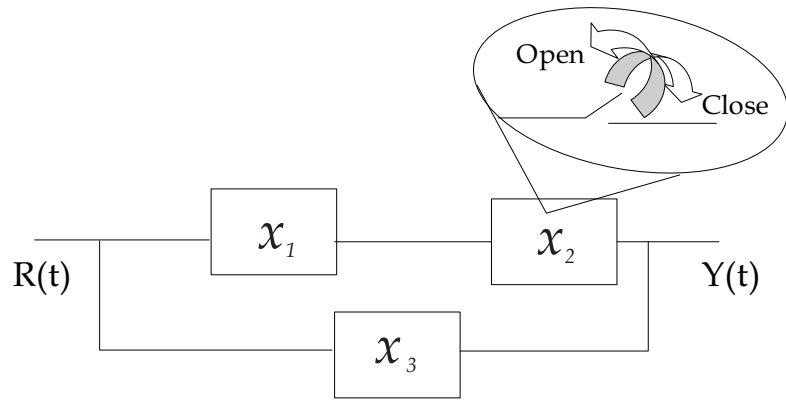

Fig. 5. Block diagram of switching element

Fig. 5 shows a switching element. For instance, a fluid flow valve and an electronic diode are two typical switching devices. Such components are multi-state because they have multiple performance levels in both modes, depending on the combination of elements available at the moment. As a result, the availability of the circuit could be defined as the probability of satisfaction of given constraints imposed on system performance in both modes (open and closed).

In this study, system availability $S_{a}(t)$ is considered to be a measure of its ability to meet the (demand) required performance level at each mode. Let $y_{p, m}(t)$ be output performances in mode $m$ of the S2FM at time $t$. Thus $y_{p, c}(t)$ and $y_{p, o}(t)$ become output performances of the system at time $t$ in its closed and open modes respectively.

Let $f_{m}\left(y_{p, m}(t), \eta_{m}\right)$ be function representing the desired relation between the system performance level and demand in mode $m$. The system fails in open mode if condition $f_{o}\left(y_{p, o}(t), \eta_{o}\right) \geq 0$ is not satisfied; it fails in the closed mode if condition $f_{c}\left(y_{p, c}(t), \eta_{c}\right) \geq 0$ is not satisfied.

Consider $P_{r}\left\{f_{m}\left(y_{p, m}(t), \eta_{m}\right)\right\}$ system failure in mode $m$. Note that, in the situation presented in Fig. 4, the occurrence probability of the failures in open and closed modes is the same for each component. This is a specific characteristic of homogeneous multi-state systems. Mathematically, a system is homogeneous when it obeys the commutative and the associative laws. As a result, because the failures in open and closed modes, which have probabilities:

$$
\left\{\begin{array}{l}
\vartheta_{o}\left(t, \eta_{o}\right)=P_{r}\left\{f_{o}\left(y_{p, o}(t), \eta_{o}\right)\right\}<0 \\
\vartheta_{c}\left(t, \eta_{c}\right)=P_{r}\left\{f_{c}\left(y_{p, c}(t), \eta_{c}\right)\right\}<0
\end{array}\right.
$$

respectively are mutually exclusive events, and the probabilities of both modes are 0.5 (each command to close is followed by command to open and vice versa), the entire system availability $S_{a}(t)$ is defined as: 


$$
S_{a}\left(t, \eta_{c}, \eta_{o}\right)=1-0.5\left(\vartheta_{c}\left(t, \eta_{c}\right)+\vartheta_{o}\left(t, \eta_{o}\right)\right)
$$

where, $\eta_{c}$ and $\eta_{o}$ are required (demand) system output performances in the system's closed and open modes respectively.

While the application of design constraints and engineering relations can occasionally yield analytical relationships which can be exploited for system safety monitoring purposes, there are no global relationships which are able to transform complex measures of performance, like cost and usability, into analytical design relations. There are no such global analytical relationships because, by their very nature, they cannot incorporate the essence of the design process, which is the use of engineering judgment to develop strategies for solving multi-objective problems.

\section{The u-function representation of system/ element performance distribution}

The system depicted in Fig. 3 is a multi-state system and the capacity or productivity of its elements is the performance measure. The problem posed by this system is one of combinational optimization. The state of the system is determined by the states of its elements. Therefore, the performance rates (levels) of the system are determined by the performance levels of its elements. As a result, the independence of the evidence to be combined would obviously be satisfied if all components' models were completely different, that is, had no overlapping equations.

A conventional controller design procedure does not guarantee those requirements and it may not even be possible to develop such a set of models. Note that the overlapping equations exist in a different environment in each model. This is sufficient for the independence of evidence, in the sense that noise and modeling errors will cause different distortions to the probability assignments in the different models.

Assume the probability distribution $\sigma_{d}$ of performance rates for all of the system elements at any instant $t \geq 0$ and system structure function as follows:

$$
\left\{\begin{array}{l}
g_{j}, p_{j} \rightarrow 1 \leq j \leq n \\
\phi\left(G_{1}(t) \ldots G_{n}\right)
\end{array}\right.
$$

In general, the total number of possible states or performance rates of the system is:

$$
\pi=\prod_{j=1}^{n} k_{j}
$$

Let $L_{n}=\prod_{j=1}^{n}\left\{x_{m, 1}, \ldots ., x_{m, k}\right\}$ be the space of possible combinations of performance rates for all system elements and $M=\left\{x_{m, 1}, \ldots, x_{m, \pi_{p}}\right\}$ be the space of possible values of entire system performance levels.

The transform $\phi\left(G_{1}(t) \ldots G_{n}(t)\right): L_{n} \rightarrow M$ which maps the space of performance rates of system elements into the space of system's performance rates, is the system structure function, [23]. 
The probability of the system to be in a given mode can be obtained as: $\sigma=\prod_{j=1}^{n} \sigma_{j, i}$; the performance rate for state $i$ is:

$$
g_{i}=\phi\left(x_{m, 1}, \ldots, x_{m, i}\right)
$$

The function $\phi($.$) is strictly defined by the type of connection between elements in the$ reliability logic-diagram sense, i.e. on the structure of the logic-diagram representing the system/subsystem. Despite the fact that the universal generating function resembles a polynomial, it is not a polynomial because: $i$.) its exponents are not necessary scalar variables, but can be arbitrary mathematical objects (e.g. vectors); ii.) the operator defined over the universal generating function can differ from the operator of the polynomial product (unlike the ordinary generating function technique, only the product of polynomials is defined) [24].

For instance, consider a flow transmission system (e.g., ore, fluid, energy) shown in Fig.4, which consist of three elements. The system performance rate which is defined by its transmission capacity can have several discrete values depending on the state of control equipments. For instance, the element 1 has three states with the performance rates $g_{1,1}=1.5$, $g_{1,2}=1, g_{1,3}=0$ and the corresponding probabilities are $\sigma_{1,1}=0.8, \sigma_{1,2}=0.1$ and $\sigma_{1,3}=0.1$. The element 2 has three states with the performance rates $g_{2,1}=2, g_{2,2}=1.5, g_{2,3}=0$ and the corresponding probabilities $\sigma_{2,1}=0.7, \sigma_{2,2}=0.22$ and $\sigma_{2,3}=0.08$. The element 3 has two states with the performance rates $g_{3,1}=4, g_{3,2}=0$ and the corresponding probabilities $\sigma_{3,1}=$ 0.98 and $\sigma_{3,2}=0.02$. According to (9) the total number of the possible combinations of the states of elements is $\pi=3 \times 3 \times 2=18$.

In order to obtain the output performance for the entire system with the arbitrary structure function $\phi($.$) , a general composition operator \partial_{\phi}$ over individual universal $z$-transform representations of $n$ system elements is defined as follows:

$$
\begin{aligned}
& \left\{\begin{array}{l}
U(z)=\partial_{\phi}\left(u_{1}(z), \ldots, u_{n}(z)\right) \\
u(z)=\sum_{i=1}^{k_{j}} \sigma_{j, i} \cdot z^{g_{j, i}}
\end{array}\right. \\
& U(z)=\sum_{i}^{k_{1}} \sum_{i}^{k_{2}} \ldots \sum_{i}^{k_{n}}\left(z^{\phi\left(x_{m, 1}, \ldots, x_{m, n}\right)} \cdot \prod_{j=1}^{n} \sigma_{j}\right)
\end{aligned}
$$

where $U(z)$ is $z$-transform representation of output performance distribution for the entire system; $u(z)$ is a polynomial $\mathrm{u}$-function of a multi-state stationary output performance.

Note that, each term of the polynomials relates probability of a certain combination of states of the subsystems to the performance level of the entire system corresponding to the combination of states defined by $\phi\left(x_{m, 1}, . . x_{m, i} . ., x_{m, n}\right)$.

Hence for a single element $i$ in mode $m$, the individual u-function is:

$$
u_{m, i}(z)=\varsigma_{a m, i} \cdot z^{x_{m, i}}+\left(1-S_{a m, i}\right) \cdot z^{\tilde{x}_{m, i}}
$$

where, $x_{m, i}$ is a nominal performance level; $\tilde{x}_{m, i}$ is fault state performance level; $S_{a m, i}$ is the availability. 
Moreover, the definition of $\phi($.$) also depends on the physical nature of system-performance$ level and on the nature of the interaction between elements. The system performance distributions in open and closed modes can be defined as follows:

$$
\left\{\begin{array}{l}
u_{o}(z)=\sum_{i=1}^{k_{j}} \sigma_{o, i} \cdot z^{y_{p 0, i}} \\
u_{c}(z)=\sum_{i=1}^{k_{j}} \sigma_{c, i} \cdot z^{y_{p c, i}}
\end{array}\right.
$$

The probability that the conditions stated in (4) are met is determined as follows:

$$
\begin{aligned}
& \left\{\begin{aligned}
\vartheta_{o}\left(t, \eta_{o}\right) & =P_{r}\left\{\mathrm{f}_{o}\left(y_{p, o}(t), \eta_{o}\right)\right\}<0 \\
& =\sum_{i=1}^{k_{j}} \sigma_{o, i} \mathrm{f}_{o}\left(y_{p, o}(t), \eta_{o}\right)<0
\end{aligned}\right. \\
& \left\{\begin{aligned}
\vartheta_{c}\left(t, \eta_{c}\right) & =P_{r}\left\{\mathrm{f}_{c}\left(y_{p, c}(t), \eta_{c}\right)\right\}<0 \\
& =\sum_{i=1}^{k_{j}} \sigma_{c, i} \mathrm{f}_{c}\left(y_{p, c}(t), \eta_{c}\right)<0
\end{aligned}\right.
\end{aligned}
$$

Thus, we could determine the anticipated performance of the system in mode $m$. Using the system's output performance distribution, the statistically expected performance in mode $m$ can be determined as:

$$
E_{m}=\sum_{i=1}^{k_{j}} \sigma_{m, i} \cdot y_{p m, i}
$$

It is very important to point out that, in the worst case, the operation time of the entire system goes to infinity. As a result, the determination of the statistically expected performance $E_{m}$ using (12) makes no sense. Therefore, the more reasonable way of evaluating the statistically expected performance is by using the statistically expected operation time in the range of its finite values (i.e., the conditional statistically expected performance, given the operation time, is finite). In this case, (12) becomes:

$$
E_{m, o p}=\frac{\sum_{i=1}^{k_{j}}\left(\sigma_{m, i} \cdot y_{p m, i}\right)}{\sum_{i=1}^{k_{j}} \sigma_{m, i}}
$$

\section{Algorithms for determining system reliability in failure modes}

In order to estimate both, systems' statistically expected operation time and its performance, different measures can be used depending on the application. The froth phase is extremely important in the operation of a flotation cell shown in fig.4, because, it is critical in determining the amount of unwanted gangue collected in the concentrate which, in turn, 
affects the purity of the product. Since the execution time of each task is of critical importance in this process, the system reliability is defined (according to performability concept) as a probability that the correct output is produced in less time than its maximal finite realization time allowed.

The above functions can be used for defining the operator for verity of configurations: series, parallel, and bridge connection of multi-state subsystems and the -functions of individual switching elements for two different types of system with two failure modes. These systems are distinguished by their specific performance measures which are: transmitted flow that characterizes performance of flow valves, and operation time that characterizes performance of electronic switches.

In general, failures reduce element performance and therefore, different performance degradation levels should be considered. This algorithm is developed to evaluate the transmitted flow and the operation time models.

To assess performance of multi-state systems when subsystems are not bridged, one should consider composition operators over pairs of u-functions corresponding to the elements connected in series and parallel and use a recursive procedure to determine the u-function of the entire series-parallel system. The following section presents algorithms for determining performance distributions and the distribution of the total execution time.

\subsection{Operation time model}

The operation time is the time between 'the instant when a command arrives to the system' and 'the instant when the command fulfillment is completed'.

In Fig. 5, systems are presented by multi-state systems for which the performance measure is characterized by the operation time. The availability assessment of this category includes control systems, and data processing systems without regard to computation time efficiency.

Consider for instance, proportional valves $\mathrm{v}_{1}$ and $\mathrm{v}_{2}$ connected in parallel within the flotation circuit. The command to open is fulfilled by the system only when it is fulfilled by both subsystems (valves). Therefore, the system operation time in the open mode is the greatest of the operation times of the subsystems. The composition operator $\phi($.$) for the open$ mode $(m=0)$ is obtained as follows:

$$
\phi_{0}\left(\eta_{o, \mathrm{v} 1}, \eta_{o, \mathrm{v} 2}\right)=\max \left(\eta_{o, \mathrm{v} 1}, \eta_{o, \mathrm{v} 2}\right)
$$

On the other hand, for pumps $\mathrm{pp}_{1}$ and $\mathrm{pp}_{2}$ connected in series the first disconnected pump disconnects the entire piping in the open mode $(m=0)$. In this case, operator $\phi_{s}($.$) for the$ open mode is:

$$
\phi_{s, o}\left(\eta_{o, \mathrm{pp} 1}, \eta_{o, \mathrm{pp} 2}\right)=\min \left(\eta_{o, \mathrm{pp} 1}, \eta_{o, \mathrm{pp} 2}\right)
$$

where the performance $\eta_{m, i}$ in mode $m$ of an element $i$ is defined as its operation time.

In closed mode $(m=c)$, the operation time of the system is the shortest of the operation times of the subsystems in parallel. Therefore, the composition operator $\phi_{p}($.$) is defined for$ valves $v_{1}$ and $v_{2}$ in parallel as follows:

$$
\phi_{p, c}\left(\eta_{c, \mathrm{v} 1}, \eta_{c, \mathrm{v} 2}\right)=\min \left(\eta_{c, \mathrm{v} 1}, \eta_{c, \mathrm{v} 2}\right)
$$


However, for pumps $\mathrm{pp}_{1}$ and $\mathrm{pp}_{2}$ connected in series, both of them should fulfill the command to close in order to make the system closed $(m=c)$.

$$
\phi_{s, c}\left(\eta_{c, \mathrm{v} 1}, \eta_{c, \mathrm{v} 2}\right)=\max \left(\eta_{c, \mathrm{v} 1}, \eta_{c, \mathrm{v} 2}\right)
$$

Consider a system with $n$ elements with total failures in open and closed modes. Assume the element operates in times $\tau_{o}$ and $\tau_{c}$ in open and closed modes respectively. If the element fails to operate, its operation time goes to infinity: $\tau \rightarrow \infty$. In parallel configuration, we have:

$$
\left\{\begin{array}{l}
u_{p, c}(z)=\left(1-\left(1-\varepsilon_{c}\right)^{n}\right) \cdot z^{\tau_{c}}+\left(1-\varepsilon_{c}\right)^{n} \cdot z^{\infty} \\
u_{p, o}(z)=\varepsilon_{o}^{n} \cdot z^{\tau_{o}}+\left(1-\varepsilon_{o}\right)^{n} \cdot z^{\infty}
\end{array}\right.
$$

where $\varepsilon_{o}$ and $\varepsilon_{c}$ are probabilities in open and closed modes respectively.

In series configuration, the u-function is given as:

$$
\left\{\begin{array}{l}
u_{s, c}(z)=\varepsilon_{c}^{n} \cdot z^{\tau_{c}}+\left(1-\varepsilon_{c}\right)^{n} \cdot z^{\infty} \\
u_{s, o}(z)=\left(1-\left(1-\varepsilon_{o}\right)^{n}\right) \cdot z^{\tau_{o}}+\left(1-\varepsilon_{o}\right)^{n} \cdot z^{\infty}
\end{array}\right.
$$

Despite composition operators are implemented for the operation time, another paradigm in multi-state system assessment is based on transmitted flow model. This is achieved according to the following stages.

\subsection{Transmitted flow model}

Transmitted flow is the amount of flow that passes through the system during each time unit. That flow is equal to the sum capacity of each subsystem:

$$
\phi_{m, k}\left(\eta_{m, 1}, \ldots . ., \eta_{m, n}\right)=\sum_{k=1}^{n} \eta_{m, k}
$$

The composition operator for the entire system is:

$$
U_{m, k}(z)=\sum_{k}^{n_{1}} \sum_{k}^{n_{2}} \ldots \sum_{k}^{n_{n}} \sigma_{m, 1} \cdots \sigma_{m, k} \bullet z^{\sum \eta_{m, 1}, \ldots \ldots, \eta_{m, n}}
$$

In series configuration, (18) becomes:

$$
\phi_{s, m}\left(\eta_{m, 1}, \ldots ., \eta_{m, n}\right)=\min \left(\eta_{m, 1}, \ldots . ., \eta_{m, n}\right)
$$

where the random performance $\eta_{m, i}$ in mode $m$ of an element $i$ is defined as its transmitting capacity, $\tau_{m}$. In the failure state it will fail to transmit any flow, $\tau_{m}=0$.

The u-function of an individual flow transmitting element in open and closed mode is defined as follows:

$$
\left\{\begin{array}{l}
u_{o}(z)=\varepsilon_{o} \cdot z^{0}+\left(1-\varepsilon_{o}\right) z^{\tau_{o}} \\
u_{c}(z)=\varepsilon_{c} \cdot z^{\tau_{o}}+\left(1-\varepsilon_{c}\right) \cdot z^{0}
\end{array}\right.
$$

where $\tau_{0}$ is the nominal flow transmitted. 
An individual flow transmitting element with total failures in the closed mode, $(m=c)$, and operational state with probability $\varepsilon_{c}$, transmits nominal flow $\tau_{0}$. In general, failures reduce system performance and, therefore, different performance degradation should be considered.

\subsection{Control valve processing speed distribution}

The electro-hydraulic valves in use in metallurgy require a reliable servo valve system tailored to provide high reliability within permissible weight and space-volume parameters. Specifically, the servo valve system includes a control system, an actuator and a main spool. If a pilot valve sticks at any position, the main stage spool of the valve could stroke unpredictably to either endpoint.

Hardware-software components are failure-prone. Their performance with regard to computation time constitutes a tradeoff between software performance and reliability. The distribution of task execution time of a control valve, such as in Fig. 4, was not explicitly determined in the above procedures.

Since the performance of the control valve depends on hardware processing speed (which in its turn depends on availability of computational resources), the impact of hardware availability should be taken into account when the system performance and availability are evaluated. Different measures are appropriate to different application areas. The reliability index we generate for control valve processing analysis can be referred to as $R(\infty)=\operatorname{Pr}\left(T_{t}<\infty\right)$ (which is equal to the probability that the random execution time, $T_{t}$, is not greater than its maximal finite realization). As a result, the conditional expected system execution time, $T_{E}$ (given the system produces correct output) is considered to be a measure of its performance. This index determines the expected execution time of the system given that the system does not fail. It can be obtained as:

$$
\left\{\begin{array}{l}
\tau_{E}=\frac{\sum_{i=1}^{n} \sum_{j=1}^{n} Q_{i, j} t_{i j}}{R(\infty)} \\
t_{i j}=\frac{h_{j}}{s_{i}}=\frac{h_{j-1}+(n-j+1)\left(c_{j}-c_{j-1}\right)}{s_{i}}
\end{array}\right.
$$

where $c_{j}$ is the computational complexity of the $j^{\text {th }}$ version; $Q_{i j}$ is the probability that the task terminates after stage $i$; $t_{i j}$ is the total time of task execution.

More importantly, note that software can have small unnoticeable errors or drifts that can culminate into a disaster. Fixing problems may not necessarily make the software more reliable. On the contrary, new serious problems may arise. Different from traditional hardware reliability, software reliability is not a direct function of time. Therefore, the distribution of the number of correct outputs after the execution of a group of first $j$ versions is given as follows:

$$
\left\{\begin{aligned}
\xi_{j}(z) & =\prod_{j=1}^{k} v_{j}(z)=\sum_{j=1}^{k} \alpha_{j} z^{j} \\
& =\xi_{j-1}(z) v_{j}(z) \\
v_{j}(z) & =a_{j} z^{1}+\left(1-a_{j}\right) z^{0}
\end{aligned}\right.
$$


The u-function which defines the performance of the processing units is:

$$
u_{i}(z)=b_{i} z^{\beta_{i}}+\left(1-b_{i}\right) z^{0}
$$

where $\beta_{i}$ is the performance of the $i^{\text {th }} \mathrm{PU}$ with probability $b_{i}$

The composition operator which defines the performance distribution of a pair of PUs is the product of the corresponding polynomials:

$$
\left\{\begin{aligned}
U(z) & =u_{i}(z) \otimes u_{j}(z) \\
& =\left[b_{i} z^{\beta_{i}}+\left(1-b_{i}\right) z^{0}\right]\left[b_{j} z^{\beta_{j}}+\left(1-b_{j}\right) z^{0}\right]
\end{aligned}\right.
$$

Using the probability mass function, $f_{m}=\sum_{j}^{n} a_{j} z^{t_{i j}}$ one could determine the task execution time distribution:

$$
\tau_{d}=f_{m}(z) \underset{\sim}{\otimes} U(z)=\sum_{i=1}^{n} \sum_{j=1}^{n} Q_{i, j} z^{t_{i j}}
$$

To use composition operators, one should find the u-function of the entire multi-state system. To do this, first determine the individual $\mathrm{u}$-functions of each element. The following algorithm is used to formulate the composition operators and determine the performance of multi-state systems.

1. Determine the u-function of each subsystem

2. Define the initial value $\xi_{0}(z)=1$ (for software reliability only)

3. Find $\mathrm{u}$-function of the entire parallel-series pair of components

4. For all versions of the software involved determine $\xi_{j}(z)$ and assign the coefficient in $\xi_{j}(z)$ to the probability, $a_{j}$, that the set of $\mathrm{k}$ version produce exactly $j$ correct outputs

Despite the operator's expertise and knowledge of the inference states of all subsystems, it is noteworthy that the reliability of the presented multi-state system is dependent on the efficiency and performance of the valves and controls involved in operating the mineral processing. Specifically, servo valves form a critical link in flow transmitting mode and a malfunction of these components is detrimental to the smooth operation of the flotation mechanism.

We had to work with the expected conditional distributions of variables associated to a specific failure mode $m$, given the values of variables associated to the preceding failure modes $(m-1)$. A direct consequence of this observation is that, one could probably improve on the reliability value by considering all $m$ ! ( $m$ factorial) possible modes and choosing the one that successfully address users needs. It is however unclear that the slight improvement achieved by that compensates for the additional computational effort is needed.

\section{Numerical example}

As above pointed out, several configurations of multiple failure mode systems could be constructed by placing the conventional single failure mode systems in a multi-state environment. For example, considering a relay circuit with a bridge structure topology and assuming that each of the components can be either "failed-open" or "failed-closed" gives birth 
to a typical multiple failure mode systems. In this sense, the well known $k$-out-of- $n$, consecutive k-out-of- $n$ systems and their generalization (the interested reader may refer to the monograph by Kuo (2003)) can be effortlessly adjusted to a multi-state environment.

In this section we shall proceed to the numerical evaluation of the proposed algorithm for dual failure mode system (S2FM). It is a series-parallel flow transmission switching system with the configuration shown in fig.4. The system is characterized by its availability and performance-level in open and close modes. For a flow transmission system, the performance of an element is its transmitting capacity, $\tau$. For a system of electronic switches, the performance of an element is determined by its operation times in open mode, $\tau_{0}$, and in closed mode, $\tau_{c}$.

In order to determine the system-performance distribution in the open and closed modes, one has to obtain the $\mathrm{u}$-function of the entire system using composition operators over $\mathrm{u}$ functions of individual elements. Consider a control hardware system consists of two PUs; software with $k=3, n=6$ (number of versions that may produce correct result and the total number of software versions, respectively). The availability, computing reliability, computational complexity of the software, speed, and parameters of the system elements are presented in Table 1. Due to operational conditions, sensors produce large amount of data to account for specific experiments, hence connectivity to computational resources is provided well in excess of normal throughput rates. Moreover, modularity is accomplished on a stage basis such that a large number of sensors are accommodated at modest overhead for scalability.

\begin{tabular}{|c|c|c|c|c|c|c|}
\hline Version : $i$ & 1 & 2 & 3 & 4 & 5 & 6 \\
\hline$c_{i}$ & 7 & 8 & 13 & 15 & 18 & 19 \\
\hline$a_{i}$ & 0.68 & 0.82 & 0.71 & 0.9 & 0.84 & 0.92 \\
\hline$b_{i}$ & 0.93 & 0.82 & \multirow{2}{*}{\multicolumn{4}{|c|}{ Parameters of PUs }} \\
\hline$\beta_{i}$ & 4 & 7 & & & & \\
\hline
\end{tabular}

Table 1. Parameter of system elements and the control software

The distribution of the system cumulative processing speed using u-function (25) is as follows:

$$
\left\{\begin{aligned}
U(z) & =u_{i}(z) \otimes u_{k}(z)=u_{1}(z) \times u_{2}(z) \\
& =\left(0.93 z^{4}+0.07 z^{0}\right)\left(0.82 z^{7}+0.18 z^{0}\right) \\
& =0.76 z^{11}+0.06 z^{7}+0.17 z^{4}+0.01 z^{0}
\end{aligned}\right.
$$

After we removed terms corresponding to total hardware failure:

$$
U(z)=0.76 z^{11}+0.06 z^{7}+0.17 z^{4}
$$

The number of computations till termination of each stage $\boldsymbol{h}_{j}$ is:

$$
h_{j}=h_{j-1}+(n-j+1)\left(c_{j}-c_{j-1}\right)
$$




$$
h_{0}=0 ; h_{1}=0+6(7-0)=42 ; h_{2}=42+5(8-7)=47
$$

Successive iteration gives: $h_{3}=67 ; h_{4}=73 ; h_{5}=79 ; h_{6}=80$;

We could use the algorithm to determine $\xi_{j}(z)$, and then define the probability $a_{j}$. Based on data provided in Table 1, we have:

$$
\begin{aligned}
& V_{0}(z)=1 ; v_{1}(z)=0.68 z^{1}+0.32 z^{0} ; V_{1}(z)=V_{0}(z) \cdot v_{1}(z) \\
& V_{2}(z)=V_{1}(z) \cdot v_{2}(z)=0.61 z^{2}+0.36 z^{1}+0.03 z^{0} \\
& V_{3}(z)=V_{2}(z) \cdot v_{3}(z)=0.5 z^{3}+0.40 z^{2}+0.09 z^{1}+0.006 z^{0}
\end{aligned}
$$

Remove the term $0.5 z^{3}$ from $V_{3}(z)$ and obtain: $a_{3}=0.5$

$$
\begin{gathered}
V_{4}(z)=V_{3}(z) \cdot v_{4}(z)=\left(0.40 z^{2}+0.09 z^{1}+0.006 z^{0}\right) v_{4}(z) \\
V_{4}(z)=0.36 z^{3}+0.08 z^{2}+0.06 z^{1}+0.1 z^{0}
\end{gathered}
$$

Remove the term $0.36 z^{3}$ from $V_{4}(z)$ and obtain: $a_{4}=0.36$; thus

$$
V_{4}(z)=0.08 z^{2}+0.06 z^{1}+0.1 z^{0}
$$

Continuously we have:

$$
\begin{array}{cc}
V_{5}(z)=V_{4}(z) \cdot v_{5}(z)=0.07 z^{3}+0.064 z^{2}+0.09 z^{1}+0.016 z^{0} ; & a_{5}=0.07 ; \\
V_{6}(z)=V_{5}(z) \cdot v_{6}(z)=0.06 z^{3}+0.09 z^{2}+0.022 z^{1}+0.001 z^{0} ; & a_{6}=0.06 ;
\end{array}
$$

Finally the probability mass distribution, $f_{m}$, is given using $a_{j}$ and $h_{j}$ :

$$
f_{m}=0.5 z^{67}+0.36 z^{73}+0.07 z^{79}+0.06 z^{80}
$$

The $\mathrm{u}$-function representing the task execution time distribution, $T_{s}$, is calculated using (26):

$$
\begin{aligned}
\tau_{s}=\left(0.5 z^{67}+0.36 z^{73}+0.07 z^{79}+0.06 z^{80}\right) \underset{\sim}{\otimes}\left(0.76 z^{11}+0.06 z^{7}+0.17 z^{4}\right) \\
\tau_{s}=0.38 z^{6.09}+0.274 z^{6.64}+0.0532 z^{7.18}+0.0456 z^{7.27}+ \\
+0.03 z^{9.57}+0.0216 z^{10.43}+0.0042 z^{11.29}+0.0036 z^{11.43}+ \\
+0.085 z^{16.75}+0.0612 z^{18.25}+0.012 z^{19.75}+0.0102 z^{20}
\end{aligned}
$$

As a result, the probability, $R(\infty)$ the system will provide a correct output is: $R(\infty)=98 \%$ Using the above result, we determine the probability the system will produce a correct output in time less than 9 seconds is: $R(9)=75.2 \%$

Note that $R(\infty)$ is determined without respect to the task execution time. Using (22), we determine the conditional expected system time, $\tau_{E}=8.6$ For practical engineering situation presented in figure 2, the system failure is defined as its inability to provide at least the required level of flow, in its closed mode, $m_{c}$, and to prevent the flow exceeding the setting point, in its open mode, $m_{0}$. The failure of the servo-valve is defined as its incapability to switch within required time, $\eta$. 


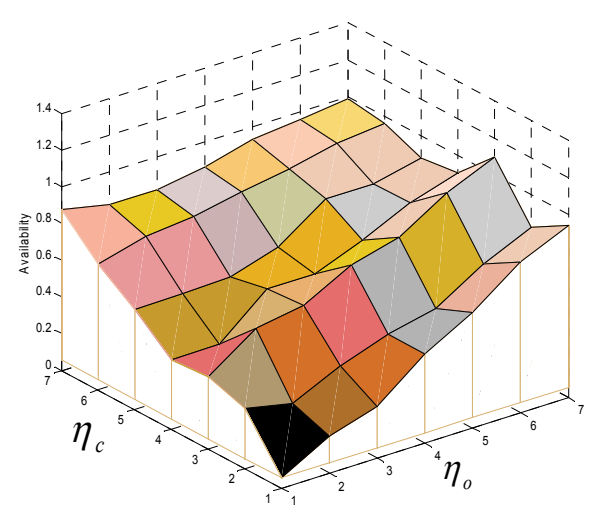

Fig. 6. Electro-valve availability $v s$ demands in open and closed modes

Fig. 6 illustrates the availability of the electro-valves as a function of required switching times, $\varsigma_{a}\left(\eta_{c}, \eta_{o}\right)$, in the open, $m_{o}$, and closed, $m_{c}$ modes. It maps the performance of the electro-valves according to their switching modes. Due to the inertia of the valves, more than $50 \%$ of their availability is reached in time $\geq 3$ seconds in either one (open or closed) mode.

The above example determined the probability that the system can produce truthful output, both without respect to the task execution time, and with task execution time less than 9 seconds respectively $R(\infty)$ and $R(9)$. Figure 7 depicts the corresponding reliability function $R\left(t^{*}\right)$ of the system to successfully execute its task in time less than $t^{*}$ for a given value of $m$ (number of versions that should produce exact results).

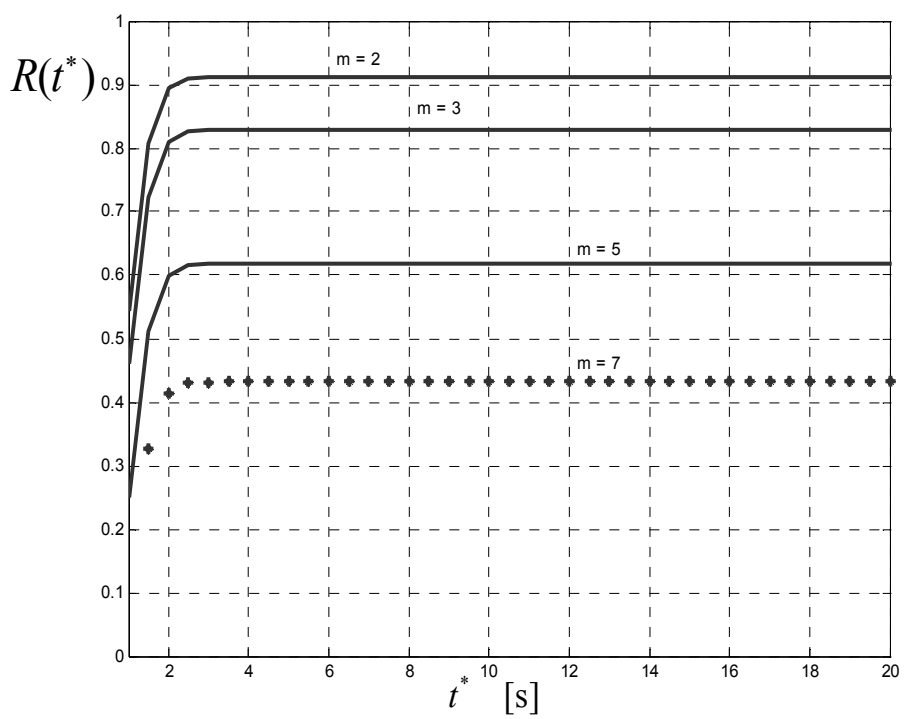

Fig. 7. Reliability function $R\left(t^{\circ}\right)$ for different values of $m$ 


\section{Conclusion}

The failure concept used in this paper is suitable for hardware and software components. It primarily deals with a broader spectrum of failures, many of which cannot be directly traced to a part failure or to any physical failure mechanism. The assumptions ignore variations in maintenance practices, load changes or additions, changing environmental conditions, circuit alterations, etc.

The developed approach can also be applied to fast evaluation of the upper bound of system performance, which is important when different system designs are compared or when system configuration optimization problems are solved in which approximate estimates of system performance should be obtained for large number of different solutions.

Although the approach does not take into consideration imperfect software task parallelization and existence of common cause failures in both hardware and software, it can be useful as a theoretical framework for in dept development of more sophisticated models.

We provided some approximate distributions for sample estimators of the measures, and approximate tests of hypotheses. Our major concerns are that, the measures of performance used by an empirical investigator should not be blindly chosen because of tradition and convention only, although these factors may properly be given some weight, but should be constructed in a manner having operational meaning within the context of the particular problem.

\section{Appendix A: Solving the optimization problem in section 2.3}

Differentiating the utilities in (6) - (8) with respect to the free choice variables when $\ell_{31}=\ell_{13}=\ell$ and $m_{k}=1$ gives the first order conditions:

$$
\begin{aligned}
& \frac{\partial u_{13}}{\partial t_{1}}=\omega_{1}\left(T_{1}+\ell T_{3}\right)\left(t_{1}+T_{1}+\ell\left(t_{3}+T_{3}\right)\right)^{-2}+\ell \omega_{3}\left(T_{3}+\ell T_{1}\right)\left(t_{3}+T_{3}+\ell\left(t_{1}+T_{1}\right)\right)^{-2}-\kappa_{1}=0 \\
& \frac{\partial u_{13}}{\partial t_{3}}=\omega_{3}\left(T_{3}+\ell T_{1}\right)\left(t_{3}+T_{3}+\ell\left(t_{1}+T_{1}\right)\right)^{-2}+\ell \omega_{1}\left(T_{1}+\ell T_{3}\right)\left(t_{1}+T_{1}+\ell\left(t_{3}+T_{3}\right)\right)^{-2}-\kappa_{3}=0 \\
& \frac{\partial u_{r 13}}{\partial T_{1}}=\varpi_{1}\left(t_{1}+\ell t_{3}\right)\left(t_{1}+T_{1}+\ell\left(t_{3}+T_{3}\right)\right)^{-2}+\ell \varpi_{3}\left(t_{3}+\ell t_{1}\right)\left(t_{3}+T_{3}+\ell\left(t_{1}+T_{1}\right)\right)^{-2}-\delta_{1}=0 \\
& \frac{\partial u_{r 13}}{\partial T_{3}}=\varpi_{3}\left(t_{3}+\ell t_{1}\right)\left(t_{3}+T_{3}+\ell\left(t_{1}+T_{1}\right)\right)^{-2}+\ell \varpi_{1}\left(t_{1}+\ell t_{3}\right)\left(t_{1}+T_{1}+\ell\left(t_{3}+T_{3}\right)\right)^{-2}-\delta_{3}=0
\end{aligned}
$$

Since are two possible decision variables, we consider the Hessian matrices. Maximum utilities exist when matrices are negative semi-define, which occurs when $\left|H_{11}\right| \leq 0$, which is satisfied, and $\left|\begin{array}{ll}H_{11} & H_{12} \\ H_{21} & H_{22}\end{array}\right| \geq 0$.

$$
H=\left|\begin{array}{ll}
\frac{\partial^{2} u_{13}}{\partial t_{1}^{2}} & \frac{\partial^{2} u_{13}}{\partial t_{1} \partial t_{3}} \\
\frac{\partial^{2} u_{13}}{\partial t_{3} \partial t_{1}} & \frac{\partial^{2} u_{13}}{\partial t_{3}^{2}}
\end{array}\right| ; \quad H_{r}=\left|\begin{array}{ll}
\frac{\partial^{2} u_{r 13}}{\partial T_{1}^{2}} & \frac{\partial^{2} u_{r 13}}{\partial T_{1} \partial T_{3}} \\
\frac{\partial^{2} u_{r 13}}{\partial T_{3} \partial T_{1}} & \frac{\partial^{2} u_{r 13}}{\partial T_{3}^{2}}
\end{array}\right|
$$


Note that: $\frac{\partial^{2} u_{13}}{\partial t_{3} \partial t_{1}}=\frac{\partial^{2} u_{13}}{\partial t_{1} \partial t_{3}}$ and $\frac{\partial^{2} u_{r 13}}{\partial T_{3} \partial T_{1}}=\frac{\partial^{2} u_{r 13}}{\partial T_{1} \partial T_{3}}$

$$
\begin{aligned}
& \frac{\partial^{2} u_{13}}{\partial t_{1}^{2}}=-2 \omega_{1}\left(T_{1}+\ell T_{3}\right)\left(t_{1}+T_{1}+\ell\left(t_{3}+T_{3}\right)\right)^{-3}-2 \ell^{2} \omega_{3}\left(T_{3}+\ell T_{1}\right)\left(t_{3}+T_{3}+\ell\left(t_{1}+T_{1}\right)\right)^{-3} \leq 0 \\
& \frac{\partial^{2} u_{13}}{\partial t_{3}^{2}}=-2 \omega_{3}\left(T_{3}+\ell T_{1}\right)\left(t_{3}+T_{3}+\ell\left(t_{1}+T_{1}\right)\right)^{-3}-2 \ell^{2} \omega_{1}\left(T_{1}+\ell T_{3}\right)\left(t_{1}+T_{1}+\ell\left(t_{3}+T_{3}\right)\right)^{-3} \leq 0 \\
& \frac{\partial^{2} u_{13}}{\partial t_{1} \partial t_{3}}=-2 \omega_{1}\left(T_{1}+\ell T_{3}\right)\left(t_{1}+T_{1}+\ell\left(t_{3}+T_{3}\right)\right)^{-3}-2 \ell \omega_{3}\left(T_{3}+\ell T_{1}\right)\left(t_{3}+T_{3}+\ell\left(t_{1}+T_{1}\right)\right)^{-3} \leq 0 \\
& \frac{\partial^{2} u_{r 13}}{\partial T_{1}^{2}}=-2 \varpi_{1}\left(t_{1}+\ell t_{3}\right)\left(t_{1}+T_{1}+\ell\left(t_{3}+T_{3}\right)\right)^{-3}-2 \ell^{2} \varpi_{3}\left(t_{3}+\ell t_{1}\right)\left(t_{3}+T_{3}+\ell\left(t_{1}+T_{1}\right)\right)^{-3} \leq 0 \\
& \frac{\partial^{2} u_{r 13}}{\partial T_{3}^{2}}=-2 \varpi_{3}\left(t_{3}+\ell t_{1}\right)\left(t_{3}+T_{3}+\ell\left(t_{1}+T_{1}\right)\right)^{-3}-2 \ell^{2} \varpi_{3}\left(t_{1}+\ell t_{3}\right)\left(t_{1}+T_{1}+\ell\left(t_{3}+T_{3}\right)\right)^{-3} \leq 0 \\
& \frac{\partial^{2} u_{r 13}}{\partial T_{1} \partial T_{3}}=-2 \ell \varpi_{1}\left(t_{1}+\ell t_{3}\right)\left(t_{1}+T_{1}+\ell\left(t_{3}+T_{3}\right)\right)^{-3}-2 \ell \varpi_{3}\left(t_{3}+\ell t_{1}\right)\left(t_{3}+T_{3}+\ell\left(t_{1}+T_{1}\right)\right)^{-3} \leq 0
\end{aligned}
$$

In order to protect the system, the approach strategically satisfies the following:

$$
\left|\begin{array}{ll}
H_{11} & H_{12} \\
H_{21} & H_{22}
\end{array}\right|=4 \omega_{1} \omega_{3}\left(1-\ell^{2}\right)^{2}\left(T_{3}+\ell T_{1}\right)\left(t_{1}+T_{1}+\ell\left(t_{3}+T_{3}\right)\right)^{-3}\left(t_{3}+T_{3}+\ell\left(t_{1}+T_{1}\right)\right)^{-3} \geq 0
$$

Mathematical manipulation of (A1) we get:

$$
\left\{\begin{array}{l}
\omega_{1}\left(T_{1}+\ell T_{3}\right)\left(t_{1}+T_{1}+\ell\left(t_{3}+T_{3}\right)\right)^{-2}=\left(\kappa_{1}-\ell \kappa_{3}\right)\left(1-\ell^{2}\right)^{-1} \\
\varpi_{1}\left(t_{1}+\ell t_{3}\right)\left(t_{1}+T_{1}+\ell\left(t_{3}+T_{3}\right)\right)^{-2}=\left(\delta_{1}-\ell \delta_{3}\right)\left(1-\ell^{2}\right)^{-1}
\end{array}\right.
$$

The above system of equation is simplified as follows:

$$
\begin{gathered}
t_{1}+\ell t_{3}=\omega_{1}\left(\delta_{1}-\ell \delta_{3}\right)\left(T_{1}+\ell T_{3}\right)\left[\varpi_{1}\left(\kappa_{1}-\ell \kappa_{3}\right)\right]^{-1} \\
T_{1}+\ell T_{3}=\omega_{1} \varpi_{1}^{2}\left(\kappa_{1}-\ell \kappa_{3}\right)\left(1-\ell^{2}\right) \times\left(\omega_{1} \delta_{1}+\kappa_{1} \varpi_{1}-\ell\left(\omega_{1} \delta_{3}+\kappa_{3} \varpi_{1}\right)\right)^{-2}
\end{gathered}
$$

Combining the above equations yields:

$$
t_{1}+\ell t_{3}=\omega_{1}^{2} \varpi_{1}\left(\delta_{1}-\ell \delta_{1}\right)\left(1-\ell^{2}\right) \times\left(\omega_{1} \delta_{1}+\kappa_{1} \varpi_{1}-\ell\left(\omega_{1} \delta_{3}+\kappa_{3} \varpi_{1}\right)\right)^{-2}
$$

Repeating the procedure above gives: 


$$
\begin{aligned}
& T_{3}+\ell T_{1}=\omega_{3} \varpi_{3}^{2}\left(\kappa_{3}-\ell \kappa_{1}\right)\left(1-\ell^{2}\right) \times\left(\omega_{3} \delta_{3}+\kappa_{3} \varpi_{3}-\ell\left(\omega_{3} \delta_{1}+\kappa_{1} \varpi_{3}\right)\right)^{-2} \\
& t_{3}+\ell t_{1}=\omega_{3}^{2} \varpi_{3}\left(\delta_{3}-\ell \delta_{1}\right)\left(1-\ell^{2}\right) \times\left(\omega_{3} \delta_{3}+\kappa_{3} \varpi_{3}-\ell\left(\omega_{3} \delta_{1}+\kappa_{1} \varpi_{3}\right)\right)^{-2}
\end{aligned}
$$

Equations (A10) and (A12) are two equations with two unknown which are solved to yield (9). Analogously, equations (A9) and (A11) are solved to yield:

$$
\begin{aligned}
T_{1} & =\omega_{1} \varpi_{1}^{2}\left(\kappa_{1}-\ell \kappa_{3}\right)\left(\omega_{1} \delta_{1}+\varpi_{1} \kappa_{1}-\ell\left(\omega_{1} \delta_{3}+\varpi_{1} \kappa_{3}\right)\right)^{-2}- \\
& -\ell \omega_{3} \varpi_{3}^{2}\left(\kappa_{3}-\ell \kappa_{1}\right)\left(\omega_{3} \delta_{3}+\varpi_{3} \kappa_{3}-\ell\left(\omega_{3} \delta_{1}+\varpi_{3} \kappa_{1}\right)\right)^{-2} \\
T_{3} & =\omega_{3} \varpi_{3}^{2}\left(\kappa_{3}-\ell \kappa_{1}\right)\left(\omega_{3} \delta_{3}+\varpi_{3} \kappa_{3}-\ell\left(\omega_{3} \delta_{1}+\varpi_{3} \kappa_{1}\right)\right)^{-2}- \\
& -\ell \omega_{1} \varpi_{1}^{2}\left(\kappa_{1}-\ell \kappa_{3}\right)\left(\omega_{1} \delta_{1}+\varpi_{1} \kappa_{1}-\ell\left(\omega_{1} \delta_{3}+\varpi_{1} \kappa_{3}\right)\right)^{-2}
\end{aligned}
$$

\section{References}

Abou S. C. "Grinding process modeling for automatic control: application to mineral and cement industries". Engineering Foundation Conferences, Delft, the Netherlands, 1997

BEA (Bureau d'enquetes et d'anlayses pour le securite de l'aviation civile). Accident on 25 July 2000 at "La Patte d'Oie" in Gonesse (95), to the Concorde, registered F-BTSC operated by Air France. Paris: Ministere de l'equipment des transportes et du logement. http://www.bea-fr.org/docspa/2000/f-sc000725pa/pdf/fsc000725pa.pdf, retrieved 07/ 2009

Billinton, R., Fotuhi-Firuzabad, M., Aboreshaid, S., "Power System Health Analysis". Reliability Engineering and System Safety, Vol. 55, Issue 1, pp.1-8,1997

Chiles, J.R., "Inviting Disaster: Lessons from the Edge of Technology". Harper Collins, New York, 2002

DeJoy, D.M., Gerson, R.R.M., Schaffer, B.S., "Safety climate: assessing management and organizational influences on safety", Professional Safety, 49 (7), 2004, pp.50-57

Guan J., Wu Y., "Repairable consecutive-k-out-of-n: F system with fuzzy states". Fuzzy Sets and Systems, vol.157, pp.121-142, 2006

Hoang Pham. "Reliability analysis for dynamic configurations of systems with three failure modes". Reliability Engineering \& System Safety, Vol.63, Issue 1, pp.13-23, 1999

Huang J., Zuo M.J., Wu Y., “Generalized multi-state k-out-of-n: G systems”, IEEE Trans. Reliability vol.49, no.1, pp.105-111, 2000

Jose E. Ramirez-Marquez, David W. Coit. "Optimization of system reliability in the presence of common cause failures". Reliability Engineering \& System Safety, Vol. 92, Issue 10, pp.1421-1434, 2007

Jussi K. Vaurio. "Uncertainties and quantification of common cause failure rates and probabilities for system analyses". Reliability Engineering \& System Safety, Vol.90, Issues 2-3, pp.186-195, 2005

Kunreuther H, Heal G. "Interdependent security". Journal of Risk and Uncertainty, vol.26 (2/3), pp.231-49, 2003 
Kuo W. and Zuo M. J., Optimal Reliability Modeling Principles and Applications: John Wiley \& Sons, 2003

Levitin G., "Universal Generating Function and its Applications", Springer, Berlin, 2005

Levitin G., Suprasad V. A. "Multi-state systems with multi-fault coverage". Reliability Engineering \& System Safety, Vol.93, Issue 11, pp. 1730-1739, 2008

Levitin G., Universal Generating Function in Reliability Analysis and Optimization, Springer-Verlag, London, 2005.

Levitin, G., Lisnianski, A. Optimizing survivability of vulnerable series-parallel multi-state systems. Reliability Engineering and System Safety 79, pp. 319-331, 2003

Lisnianski A. and Levitin G., Multi-State System Reliability Assessment, Optimization, Applications: World Scientific, 2003.

Ross S.M., "Introduction to Probability Models", Academic Press, 1993

Tavakkoli-Moghaddam R., Safari J., Sassani F. "Reliability optimization of series-parallel systems with a choice of redundancy strategies using a genetic algorithm". Reliability Engineering \& System Safety, Vol.93, Issue 4, pp.550-556, 2008

Terje Aven. "On performance measures for multistate monotone systems". Reliability Engineering \& System Safety, Vol.41, Issue 3, pp.259-266, 1993

Turner, B.M., Pidgeon, N., "Man-Made Disasters", 2nd edition, Butterworth-Heinemann, London, 1997

Ushakov L.A., "Universal generating function", Sov. J. Comp. System Science, vol.24, no.5, pp.118-129, 1986

Vaughan, D. "The Challenger Launch Decision: Risky Technology Culture and Deviance at NASA", University of Chicago Press, Chicago, 1996

Xue J., Yang K., "Symmetric relations in multi-state”, IEEE Trans. on reliability, vol.44, no.4, pp.689-693, 1995

Zaitseva E. "Dynamic reliability indices for multi-state system", J. of dynamic system \& geometric theories, vol.1, no.2, pp.213-222, 2003,

Zhigang Tian, Gregory Levitin, Ming J. Zuo . "A joint reliability-redundancy optimization approach for multi-state series-parallel systems". Reliability Engineering \& System Safety, Vol.94, Issue 10, pp.1568-1576, 2009 


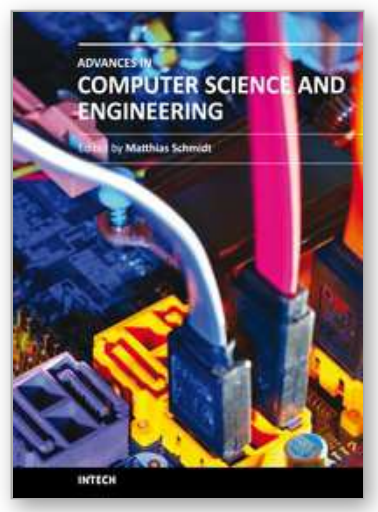

\author{
Advances in Computer Science and Engineering \\ Edited by Dr. Matthias Schmidt
}

ISBN 978-953-307-173-2

Hard cover, 462 pages

Publisher InTech

Published online 22, March, 2011

Published in print edition March, 2011

The book Advances in Computer Science and Engineering constitutes the revised selection of 23 chapters written by scientists and researchers from all over the world. The chapters cover topics in the scientific fields of Applied Computing Techniques, Innovations in Mechanical Engineering, Electrical Engineering and Applications and Advances in Applied Modeling.

\title{
How to reference
}

In order to correctly reference this scholarly work, feel free to copy and paste the following:

Seraphin C. Abou (2011). Performance Assessment of Multi-State Systems with Critical Failure Modes: Application to the Flotation Metallic Arsenic Circuit, Advances in Computer Science and Engineering, Dr. Matthias Schmidt (Ed.), ISBN: 978-953-307-173-2, InTech, Available from:

http://www.intechopen.com/books/advances-in-computer-science-and-engineering/performance-assessmentof-multi-state-systems-with-critical-failure-modes-application-to-the-flotati

\section{INTECH}

open science | open minds

\section{InTech Europe}

University Campus STeP Ri

Slavka Krautzeka 83/A

51000 Rijeka, Croatia

Phone: +385 (51) 770447

Fax: +385 (51) 686166

www.intechopen.com

\section{InTech China}

Unit 405, Office Block, Hotel Equatorial Shanghai

No.65, Yan An Road (West), Shanghai, 200040, China 中国上海市延安西路65号上海国际贵都大饭店办公楼 405 单元

Phone: +86-21-62489820

Fax: $+86-21-62489821$ 
(C) 2011 The Author(s). Licensee IntechOpen. This chapter is distributed under the terms of the Creative Commons Attribution-NonCommercialShareAlike-3.0 License, which permits use, distribution and reproduction for non-commercial purposes, provided the original is properly cited and derivative works building on this content are distributed under the same license. 